\title{
Un accès inégal à la chirurgie de la douleur
}

\section{Inequality of Access to Pain Surgery}

\author{
M. Lévêque \\ (C) Lavoisier SAS 2015
}

En 1939 - à la veille d'une période où les circonstances allaient multiplier si durement les douleurs des hommes René Leriche s'indignait : "L'histoire de bien des méthodes nous apprend que, souvent, les médecins praticiens ont compris plus vite l'intérêt des faits nouveaux que ceux qui ont pour mission de les enseigner. L'effort d'initiation doit porter plus sur l'esprit des méthodes que sur la technique. Il n'est pas nécessaire que tout le monde sache faire une infiltration stellaire. Mais il importe que tout médecin sache ce que l'on peut en attendre. Un malade ne doit pas continuer de souffrir pour la seule raison que celui qui le soigne ne sait pas qu'on peut le soulager ». Par ces mots de préface, Leriche justifiait le travail d'enseignement qui serait sien au travers de «La Chirurgie de la Douleur ». Curieusement, et réjouissons-nous en, l'ouvrage du chirurgien strasbourgeois aura davantage contribué à sensibiliser les médecins à la prise en charge de la douleur — ce « sinistre cadeau qui diminue l'homme » — qu'à faire connaître les gestes chirurgicaux en question.

Soixante-dix ans plus tard, l'éventail de ces techniques s'est considérablement étoffé et rares sont les douleurs pharmacorésistantes qui ne peuvent en bénéficier, parfois avec de spectaculaires résultats.

Pourtant, aujourd'hui encore, il est des malades qui continuent de souffrir parce que celui qui le soigne ne sait pas qu'on peut le soulager... C'est ce pénible constat qui nous a incités à publier un nouvel opus ${ }^{1}$ à l'ouvrage de Leriche et la raison d'être du numéro que vous tenez entre vos mains.

Il est regrettable, en 2015, que l'accès à ces gestes varie considérablement d'un centre à l'autre. À l'inverse des molécules, qui connaissent une diffusion beaucoup plus homogène - à laquelle l'industrie pharmaceutique n'est pas étrangère - , l'accès à ces interventions portant sur la dou-

\footnotetext{
M. Lévêque $(\square)$

Service de neurochirurgie, hôpital de la Pitié-Salpêtrière, 47-83, boulevard de l'Hôpital, F-75013 Paris, France

e-mail : marc.leveque@aphp.fr
}

1. Chirurgie de la douleur, ouvrage collectif écrit par soixante experts francophones. Éditions Lavoisier, 2014. leur est très inégal. Cela implique le fameux « effort d'initiation » cher à Leriche — et c'est là une responsabilité universitaire qui ne semble pas toujours honorée - ainsi que l'accès géographique à une structure offrant ce type de geste. Cette différence entre le médicament et l'intervention confine presque à l'allégorie avec, d'un coté, la molécule qui diffuse dans l'organisme comme sur le territoire et, de l'autre, l'intervention — focale par essence —, réservée à certaines structures...

Aujourd'hui, probablement en raison d'un défaut d'information, la stimulation médullaire et l'infusion intrathécale appartiennent à ces gestes dont l'accès est extrêmement inégal. Pour tenter de réparer cela, nous avons laissé la parole à l'équipe de Philippe Rigoard qui, dans « La stimulation médullaire en 2015 », nous détaille les indéniables avantages de cette technique dans un large spectre de douleurs neuropathiques périphériques.

Également sous-utilisée, en dépit de son intérêt majeur dans les douleurs cancéreuses mal contrôlées, Denis Dupoiron nous parle de « L'infusion intrathécale dans les douleurs irréductibles $\gg$.

La névralgie faciale lorsqu'elle ne répond plus aux médicaments, et notamment à la Carbamazépine, pose la délicate question du recours à la chirurgie. Cette mise au point sur le « Traitement de la névralgie trigéminale essentielle par décompression vasculaire microchirurgicale » par Marc Sindou nous éclaire sur les indications et les conditions du geste.

Les contre-indications à cette dernière intervention laissent une place à d'autres techniques. L'équipe de Jean Régis dans « La radiochirurgie dans le traitement de la douleur » nous décrit les avantages de l'une de ces alternatives et l'intérêt, grandissant, de cette technique stéréotaxique dans d'autres type de douleurs.

Enfin, avec les « Traitements médicamenteux et chirurgicaux de l'algie vasculaire de la face », abordés par Dominique Valade et Denys Fontaine, nous sortons du cadre strict de la technique chirurgicale pour aborder le traitement de cette pathologie douloureuse de la face dans sa globalité. 
D'aucuns jugeront le panorama de ce numéro sur la chirurgie de la douleur incomplet. Ils auront raison. Entres les réponses à nos lettres d'invitation et la sortie de ce numéro est venue s'interposer la période d'un repos estival bien mérité. Alors, doit-on en tenir grief à ceux qui s'adonnent à cette chirurgie exigeante pour laquelle, comme nous le rappelle Leriche, "l'on doit s'adapter à la désillusion de l'opéré, lui redonner confiance dans une tentative ultérieure, lui insuffler une nouvelle espérance, bref, savoir exercer l'action morale nécessaire. Les chirurgiens brusques, emportés, peu maîtres de leurs réactions, ne doivent pas s'adonner à une chirurgie qui exige un constant tonus moral, et réclame souvent la maîtrise de ses déceptions. Il faut de la diplomatie dans la chirurgie de la douleur $\gg$. Parfois en faut-il aussi pour conclure un éditorial... Bonne lecture! 